\title{
A Block-based 3D Map: a Crude Sketch of Steric Environment
}

\author{
Jongsu Yi and JunSeong Kim \\ School of Electrical and Electronics Engineering ,Chung-Ang University \\ 221 Heukseok-dong, Dongjak-gu, Seoul, 156-756 Korea \\ junkim@cau.ac.kr
}

\begin{abstract}
A 3D map provides useful information for various services. Traditionah 30 maps, however, consist of a raw image data and are not suitable for real-time applications. In this paper, we propose a block-based 3D map, which forms three dimensional spaces in acollection of cubic blocks. The block-based 3D map has two major pafameters: an object yatio and a block size. The object ratio is defined as the rate of object pixels to air-filled plxels within a block and determines the type of a block. The block size determines actual length of the side of a cubic block and is a matter of resolution and focaldistance of images. Experimental results show benefits of the block-based 3D map in redueing sensitivity to noise and in saving amounts of data to be processed. Block-based 3D maps would cupport a variety of new services by providing additional information to image data in intelligent real-time applications.
\end{abstract}

Keywords: block-based 3D map, smart cawera, stereo vision, intelligent services

\section{Introduction}

Intelligent services like a smart gyeen home, smart surveillance, and ubiquitous robotic companion continues to be a groving industry area $[1,3]$. Sensors are one of the most essential components for intelligent services since sensors collect information and directly dictate quality of the service. Traditional sensors like infrared light, heat probe, humidity sensor and so on are passive and low-level ones. For the increasing quality and complexity of intelligent services hoyever, there have been increasing demands on new types of sensor, which can recognize situations and make decisions [1, 2, 9]. Vision sensors provide rich sources of information in applications including robots, factory automations, intelligent vehicles, and home networks. Three-dimensional maps of environment, especially, can provide much more complete information. For flexible services in intelligent environments, 3D naps with value added data would be desirable. Many traditional researches on 3D maps, however, are not appropriate for real-time services since images consist of large amount of data and takes time to be manipulated $[6,8]$.

This paper describes an augmented 3D map, which forms three dimensional spaces in a collection of cubic blocks. The block-based 3D map has two major parameters: an object ratio and a block size. The object ratio is defined as the rate of object pixels to air-filled pixels within a block and determines the type of a block. The block size determines actual length of the side of a cubic block and is a matter of resolution and focal distance of images. Experiments with a 320x 240 pixel image show that the block size of $40 \times 40$ and the object ratio of $30 \%$ to $50 \%$ results in good performance. From the experimental results we can see benefits of the block-based 3D map in reducing sensitivity to noise and in saving amounts of 
data to be processed. Block-based 3D maps would support a variety of new services by providing additional information to image data in intelligent real-time applications.

The rest of the paper is organized as follows. In Section 2, we present the concepts of block-based 3D maps. In Section 3, we evaluate the effectiveness of the block-based 3D map by measuring the amount of image data and the sensitivity to noise with varying parameter values. In Section 4, we summarize the experimental results and conclusions.

\section{A block-based 3D map}

A block-based 3D map is a kind of augmented images, which consist of fixed size cubes [7]. Figure 1 shows the conceptual view of a block-based 3D map. Living space may contain various objects including a table, men, etc. We project the space into a two dimensional image by taking a snapshot in a single direction. The two-dimensional image reflects distance information being in perspective. That is, an object in far from the vanishing point appeared to be in small and an object in near to be in large. We, however, cannot restore the distance information from the 2-D image. When we have two or more images taken from different viewpoints the 3-dimensional structure can be extracted Depth foaps are the output image of stereo matching techniques. Block-based 3D maps are built by shing the original living space into cubes according to the information of the corresponding depth maps.

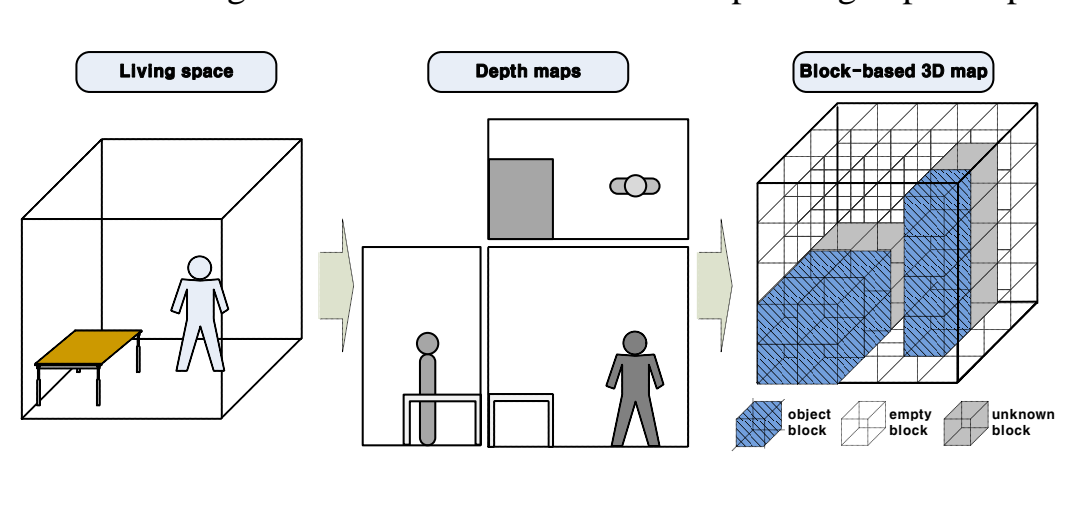

Figure 1. A conceptual view of a block-based 3D map

There are three different kinds of cubic blocks; object blocks, empty blocks, and unknown blocks. If a cubjc space contains a certain solid object we call it an object block. If there is no solid object in an air-filled cubic space we call it an empty block. Otherwise, we call a cubic space an unknown block. Unknown blocks include ones right behind an object block such that we cannot tell if there are any objects in the condition. By taking multiple snapshots of living space in different directions we can eliminate those unknown blocks.

There are two main parameters in constructing a block-based 3D map; a block size and an object ratio. A block size determines actual length of the side of a cubic block. For a given image it is a matter of resolution and focal distance. With a small block size a map can present living space accurately but has to process that much amount of data. With a large block size a map provides a crude sketch of living space but can be processed easily in realtime. A block size can be set according to applications' demands on granularity. An object ratio is defined as the rate of objects to that of emptiness within a facet of cubic blocks. When a cubic block contains a boundary of an object we may consider that the block is either full or empty depending on the object ratio. It provides the threshold between an object block and an empty block. With low object ratio a map is built in conservative way while with high object ratio it provides only a rough outlines of objects. 


\section{Experiments}

Block-based 3D maps are augmented images. While the attributes of an image depend on its applications we can simply count the size of image data for comparison. Figure 2 shows the reduction rate in image data size of a block-based 3D map for a given 320x240 pixel image. The $\mathrm{X}$-axis represents the block size in pixel. The primary $\mathrm{Y}$-axis represents reduction rate in percentage for the line graph and the secondary Y-axis represents the number of pixels for the bar graph. For example, when the block size of $5 \times 5$ pixels is in use, an image of $320 \times 240$ can be redrawn by $64 \times 48$ square blocks. This saves huge amount of image data, about $96 \%$ in reduction rate, at the cost of resolution. The larger in block size, the higher in reduction rate. We expect that these reductions in image data, in turn, can directly increase the processing speed of the images in real-time intelligent services.

\section{Figure 2. A simple comparison of image data usage in block-based 3D maps}

Figure 3 shows a sample lesulting depth map of stereo correlation. Pairs of images consisting of $320 \times 240$ pixels are used as test inputs; A cup is placed on a partition and bookshelves are ignored as background in the correlation process since it is beyond the range of consideration. We use SAD (sum of absolute differences) algorithm since it is simple and has linear data flow patterns to be processed all in parallel $[4,5]$. For the experiments we set the maximum disparity to 128 with the window size of $3 \times 3$. From the comparison of the depth map with the ideatone we can easily see noises.

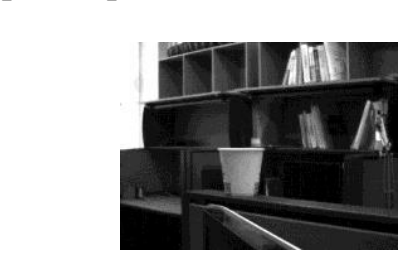

(a) left image

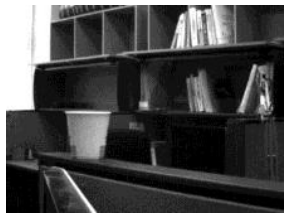

(b) right image

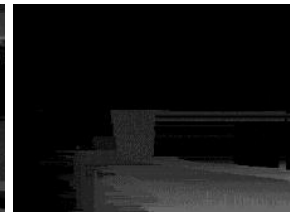

(c) depth map

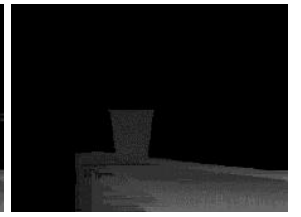

(d) ideal depth map

Figure 3. A sample depth map using SAD correlation algorithm

Figure 4 shows sample block-based 3D maps of various block sizes. Object ratio is fixed to $50 \%$. The noises in the depth map turn up erroneous blocks in block-based 3D maps. In the figure, erroneous object blocks, which are supposed to be non-object blocks, are shown in red and erroneous empty blocks, which are supposed to be non-empty blocks, are shown in blue. Since the experimental sample maps are built with only a single directional pair of stereo images there is no unknown blocks shown in the figure. Figure 5 shows the error rate, which is about the correctness of a block-based 3D map, against the block size. It is defined to be the ratio of the number of erroneous blocks to the total number of blocks in use. We can see that 
there is an optimal value in block size with respect to error rate. In this test case, 40x40 block size results in the best performance. There is a trade-off between block size and the sensitivity to noise. Images can be presented in more detail with smaller block sizes but become more sensitive to noises. Images with larger block sizes can hide noises but cannot depict in minute details. With large block sizes, also, the number of blocks used to present an image is decreased such that any single erroneous block can drastically increase the error rate. In this test case, the block size of $80 \times 80$ seems to be too large to depict the cup on a partition.

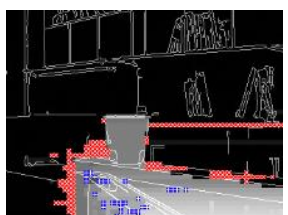

(a) $5 \times 5$

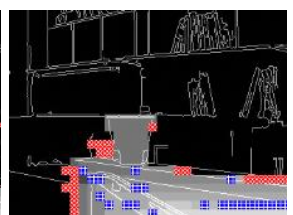

(b) $10 \times 10$

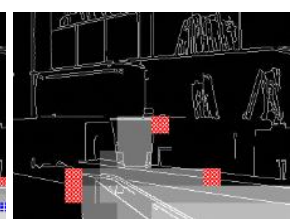

(c) $20 \times 20$

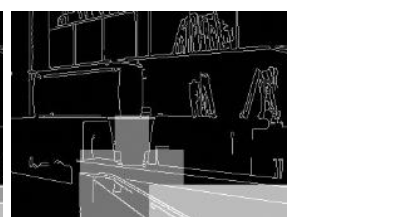

(d) $40 \times 40$

Figure 4. Block-based 3D maps with various block sizes

Figure 5. Errôrates of block-based 3D maps against block sizes

Figure 6 shows sample block-based 3D maps of various object ratios and Figure 7 shows the corresponding error rate. The block size is fixed to $40 \times 40$ pixels. We can see that there is an optimal range in object ratio with respect to error rate. In this test case, object ratio between $30 \%$ and $50 \%$ result in the best performance. The performance degradation with the object ratio outsider the optimal range results mainly from the noises in depth map. We can adjust the object ratio depending on applications' demand for levels of granularity.

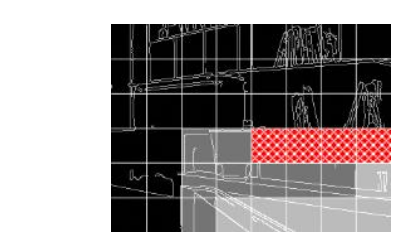

(a) $10 \%$

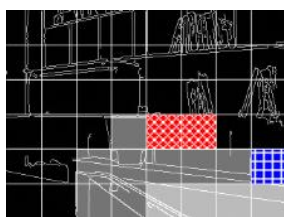

(b) $20 \%$

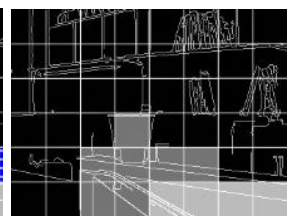

(c) $40 \%$

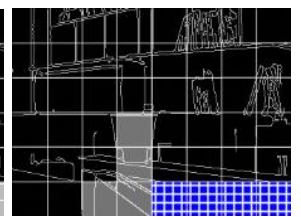

(d) $80 \%$

Figure 6. Block-based 3D maps with various object ratios 


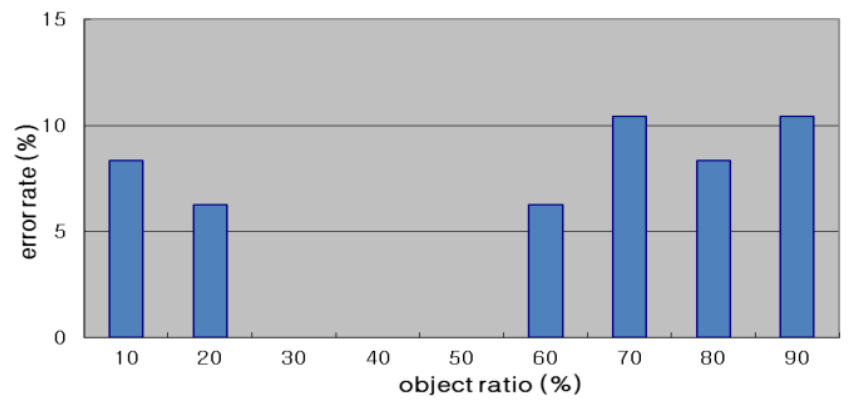

Figure 7. Error rates of block-based 3D maps against object ratios

\section{Conclusions}

A 3D map provides useful information for various intelligent services Traditional 3D maps, however, consist of a raw image data and are nonsuitable for real-ime applications. This paper presented a block-based 3D map, which forms three dimensional spaces in a collection of cubic blocks. From experimental results we saw the benefits of the block-based 3D map in reducing sensitivity to noise and in saving amounts of data to be processed. Blockbased 3D maps would support a variety of new services by providing additional information to image data in intelligent real-time applications.

\section{Acknowledgements}

This research was supported by Bdsic Science Research Program through the National Research Foundation of Korea(NRF) funded by the Ministpy of Education, Science and Technology(grant number 2013-025387). A prelinimary version of this work was presented in the Journal of the Institute of Electronics Engineers of Korea 2012 [7].

\section{References}

[1] Y. Zhang, Y. Gu, V. Vlatkdvic and X. Wang, "Progress of smart sensor and smart sensor networks", Proceedings of the Fifth World/Congress on Intelligent Control and Automation, (2004) June 15-19.

[2] P. Chen, K. Hong, N. Naikgl, S. Sastry, D. Tygar, P. Yan, A. Yang, L. Chang, L. Lin, S. Wang, E. Lobaton, S. Oh and P. Ahammad, A Low-Bandwidth Camera Sensor Platform with Applications in Smart Camera Networks", ACM Transactions on Sensor Networks, vol. 9, no. 2, (2013).

[3] J. Gubbia, R. Buyyab, S. Marusic and M. Palaniswami, "Internet of Things: A vision, architectural elements, and future dinections", Future Generation Computer Systems, vol. 29, (2013), pp. 1645-1660.

[4] S. T. Barnary and M. A. Fischler, "Computational Stereo", ACM Computing Surveys, vol. 14, no. 4, (1982), pp. $553-572$.

[5] B. Tippetts, D. J. Lee, K. Lillywhite and J. Archibald, "Review of stereo vision algorithms and their suitability for resource-limited systems", Journal of Real-Time Image Processing, (2013).

[6] J. Yi and J. Kim, "Heuristic Designs of SAD Correlation Algorithm for Vision System", Journal of the Institute of Electronics Engineers of Korea - CI, vol. 47, no. 5, (2010), pp. 61-66.

[7] J. Yi and J. Kim, "A Block based 3D Map for Recognizing Three Dimensional Space", Journal of the Institute of Electronics Engineers of Korea-CI, vol. 49, no. 4, (2012), pp. 89-96.

[8] A. Cappalunga, S. Cattani, A. Broggi, M. S. McDaniel and S. Dutta, "Real time 3D terrain elevation mapping using ants Optimization Algorithm and stereo vision", Proceedings of the IEEE Intelligent Vehicles Symposium (IV), (2010) June 21-24; San Diego, U.S.

[9] J. Sun, B. Jeon, J. Lim and M. Lim, "Stereo vision based 3D modeling system for mobile robot", Proceedings of the International Conference on Control Automation and Systems, (2010) October 27-30; Gyeonggi-do, Korea. 


\section{Authors}

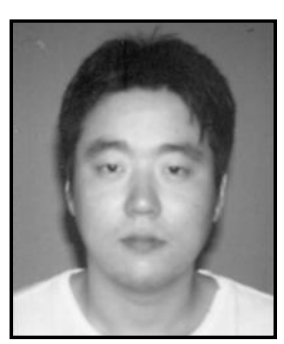

\section{Jongsu Yi}

He received a Ph.D. in Electronics Engineering from the Chung-Ang University in Seoul, Korea. His research interests include computer architecture, intelligent home service and stereo vision system. He is a member of the IEICE and a member of the IEEK.

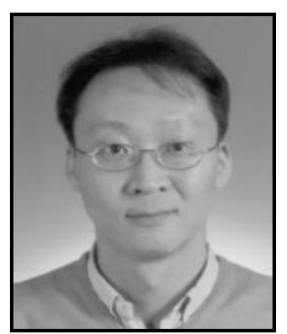

\section{JunSeong Kim}

He received a Ph.D. in Electrical Engineering from the University of Minnesota at Minneapolis, a M.S and a B.S. both in Electronics Engineering from the Chung Ang University in Seoul, Korea. He is currently a Professor of School of Electrical and Electronics Engineering at the Chung-Ang University. His main research interests include computer arehitecture, high-performance computing, parallel processing, and embedded system design.

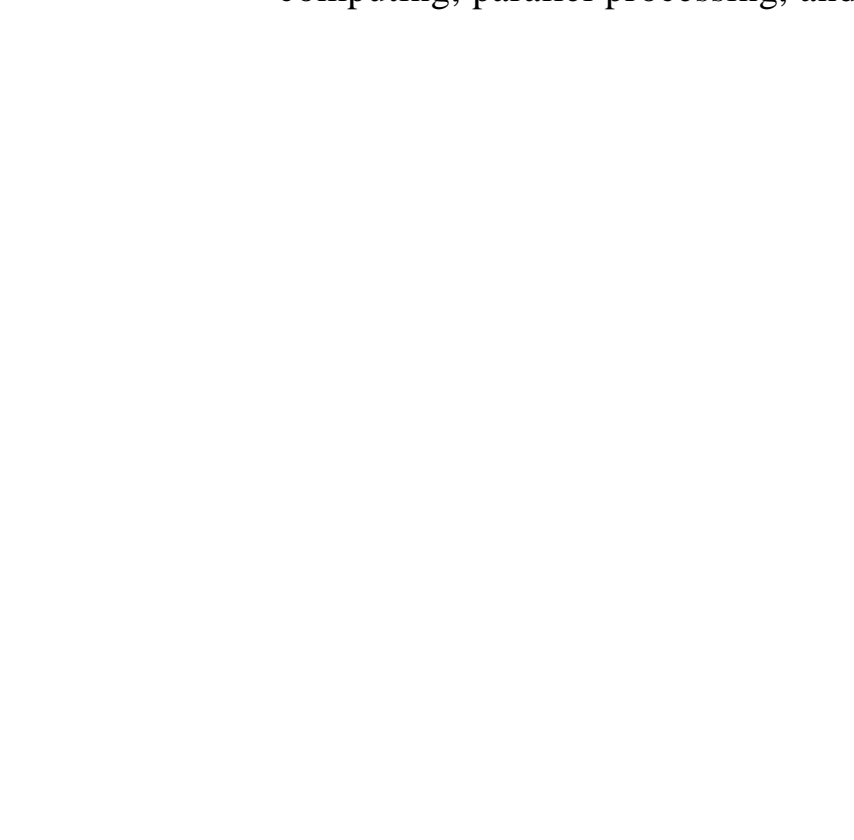

